\title{
Effects of xipayi mouth rinse combined with minocycline on localized aggressive periodontitis' therapeutic effect and the levels of CRP, TNF- $\alpha$, IL- 6 in serum
}

\author{
Xian Chen ${ }^{1}$, Li Wang ${ }^{2}$, Zhifang He ${ }^{3}$, Haijiao Zhao ${ }^{1}$, Yehua Cai ${ }^{3}$, Shanglin Song ${ }^{4}$, Qin Yu ${ }^{1}$ \\ ${ }^{1}$ Department of Prosthodontics, Handan Stomatological Hospital, Handan, China; ${ }^{2}$ Department of Pediatric Dentistry, Handan Stomatological \\ Hospital, Handan, China; ${ }^{3}$ Department of Periodontology, Handan Stomatological Hospital, Handan, China; ${ }^{4}$ Department of Clinical Laboratory, \\ Handan Stomatological Hospital, Handan, China \\ Contributions: (I) Conception and design: Q Yu, X Chen; (II) Administrative support: Q Yu; (III) Provision of study materials or patients: H Zhao, Y \\ Cai, S Song; (IV) Collection and assembly of data: L Wang, Z He; (V) Data analysis and interpretation: X Chen, L Wang, Z He; (VI) Manuscript \\ writing: All authors; (VII) Final approval of manuscript: All authors. \\ Correspondence to: Qin Yu; Xian Chen. Department of Prosthodontics, 197 Guangming Nan Dajie, Handan, China. \\ Email: 623878024@qq.com; fernandes2010@163.com.
}

Background: Localized aggressive periodontitis is rare periodontitis in clinical practice, which often occurs in young adults under 35 years old, seriously affecting patients' quality of life. As a tetracycline antibacterial drug, minocycline is also considered an essential choice to treat periodontal disease. However, few reports focused on the effect of xipayi mouth rinse combined with minocycline on periodontal pathogens. The goal of this study was to investigate the clinical effect of xipayi mouth rinse combined with minocycline in the treatment of localized aggressive periodontitis and its effect on the levels of CRP, TNF- $\alpha$, and IL-6.

Methods: Ninety-six patients with limited aggressive periodontitis were selected and randomly divided into two groups. Forty-eight patients in the control group were treated with xipayi mouth rinse after primary periodontal treatment. Then, 48 patients in the experimental group were treated with xipayi mouth rinse combined with minocycline after primary periodontal treatment. The periodontal probe was applied to detect periodontal plaque index (PLI), periodontal pocket depth (PD), sulcus bleeding index (SBI), gingival index (GL), and clinical attachment loss (CAL) before and after treatment in both groups of patients. ELISA was used for detecting the expression levels of CRP, TNF- $\alpha$, and IL-6 in the serum of patients in two groups before and after treatment. We compared the recurrence rates of the two groups after a 1-year follow-up.

Results: Compared with the control group, the PLI, PD, SBI, GL, CAL, and total masticatory efficiency of the experimental group were significantly better than those of the control group. The levels of inflammatory factors CRP, TNF- $\alpha$, and IL-6 were significantly declined, and the total effective rate of treatment was significantly elevated. After follow-up, it was found there was no noticeable difference in the recurrence rate between the two groups.

Conclusions: Xipayi mouth rinse, combined with minocycline in the treatment of localized aggressive periodontitis, can significantly improve the periodontal gingival condition and reduce the level of inflammatory factors. Also, the efficacy of the treatment was significant. This experiment has provided ideas for improving the clinical treatment of patients with localized aggressive periodontitis.

Keywords: Localized aggressive periodontitis; xipayi mouth rinse; minocycline; periodontal gingival condition; inflammatory factor

Submitted Aug 05, 2020. Accepted for publication Sep 11, 2020.

doi: 10.21037/apm-20-1653

View this article at: http://dx.doi.org/10.21037/apm-20-1653 


\section{Introduction}

Localized aggressive periodontitis is one of the most common diseases and frequently-occurring diseases in the oral cavity, which mainly occurs in teenagers, and develops rapidly. Rapid destruction of periodontal tissue and rapid resorption of alveolar bone is the primary clinical manifestation of it, which can cause tooth loosening and displacement, and even tooth loss (1-3). It mainly occurs in incisors and first permanent molars, and is generally symmetrically distributed. Its incidence is lower than that of extensive aggressive periodontitis, but it can also affect the surrounding teeth if it cannot be treated in time and effectively (4). At present, the pathogenesis of localized invasive periodontitis is not clear. Most scholars believe that plaque microbial infection plays a major role in the occurrence and development of localized invasive periodontitis. Therefore, removal of plaque microorganisms is the key to the treatment of localized invasive periodontitis (5). Antibacterial medicine combined with primary periodontal treatment was applied to treat localized aggressive periodontitis in modern medicine, but it cannot eliminate bacteria, and it is prone to produce drug-resistant strains, making treatment more difficult (6). In Chinese medicine, modern periodontal disease has long been recorded as "toothache" and "gingival atrophy," and it emphasized the main factors for the occurrence of this disease are deficiency of kidney qi, insufficiency of qi and blood, and exuberance of stomach fire. Paying attention to cutting pathogens and strengthening vital qi in treatment can effectively enhance the ability of systemic immune regulation of patients and is beneficial to the repair and reconstruction of damaged periodontal tissue. The study has observed the efficacy of xipayi mouth rinse combined with minocycline in the treatment of localized aggressive periodontitis and its effect on plaque index (PLI) and serum levels of C-reactive protein (CRP), tumor necrosis factor- $\alpha$, (TNF- $\alpha$ ) and interleukin-6 (IL-6). We present the following article in accordance with the CONSORT reporting checklist (available at http://dx.doi.org/10.21037/apm-201653).

\section{Methods}

\section{General data}

Ninety-six patients with localized aggressive periodontitis treated in the department of stomatology of our hospital from January 2018 to October 2018 were selected as the object of study. The diagnosis of western medicine accorded with the diagnostic criteria of localized aggressive periodontitis in the new classification of Armitage [1999] (7). Inclusion criteria: (I) the diagnosis of localized aggressive periodontitis was confirmed by periodontal examination; (II) all patients met the diagnostic criteria of the Armitage [1999] new classification; (III) they had not received periodontal treatment for one year before the start of the investigation; (IV) they had not been treated with antibiotics within three months; (V) they had at least 18 fully erupted teeth; (VI) all patients voluntarily participated to participate in the study and had signed an informed consent form. Exclusion criteria: (I) patients with severe systemic disease; (II) patients with dental caries simultaneously; (III) people allergic to drugs related to this study; and (IV) women during pregnancy and lactation. A random number table was used to divide all study subjects into a control group $(\mathrm{n}=48)$ and an experimental group $(\mathrm{n}=48)$. The control group was given oral treatment with xipayi mouth rinse only after primary periodontal treatment, and the experimental group was given both xipayi mouth rinse and minocycline simultaneously after primary periodontal treatment. All procedures performed in this study involving human participants were in accordance with the Declaration of Helsinki (as revised in 2013). The study was approved by the Ethics Committee of Handan Stomatological Hospital and informed consent was taken from all the patients.

\section{Therapeutic methods}

Both groups were given routine primary treatment by the same physician, including subgingival scraping and root planing under local anesthesia, cleaning the affected teeth, and surrounding gingiva, rinsing the periodontal pockets with $0.9 \%$ normal saline and $3 \%$ hydrogen peroxide, performing dental isolation techniques and blow-drying together. Both the control group and the experimental group were given routine primary treatment, including subgingival scraping and root planing under local anesthesia, cleaning the affected teeth, and surrounding gingiva to remove periodontal calculus and plaque, and rinsing periodontal pockets with $3 \%$ hydrogen peroxide and $0.9 \%$ normal saline. In the control group, patients were given only oral treatment with xipayi mouth rinse at the first dose of $5 \mathrm{~mL} /$ time-based on primary periodontal therapy. Then, it was followed by $3 \mathrm{~mL} \mathrm{~g} /$ time, three times a day, for three days. Patients in the experimental group were injected with $2 \%$ minocycline hydrochloride ointment 

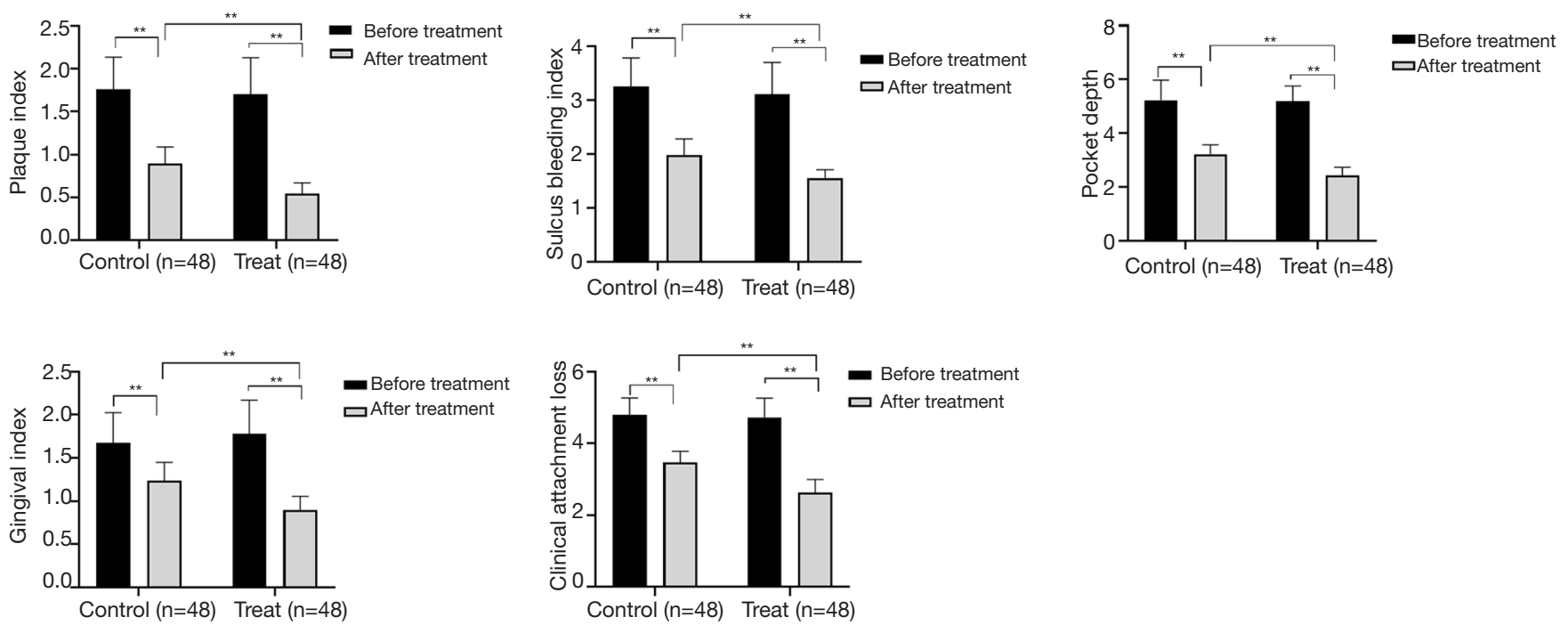

Figure 1 Comparison of periodontal indexes between the two groups before and after treatment. ${ }^{* *}, \mathrm{P}<0.01$.

into the periodontal pocket until full at periodontal probing depth $>5 \mathrm{~mm}$ sites. Patients were recommended not to eat and gargle within 1 hour, once a week, for 4 weeks. Together, the patient was given oral treatment with xipayi mouth rinse.

\section{Observational index}

(I) Clinical efficacy: the relevant standards in the "Guidelines for the Diagnosis and Treatment of Oral Diseases" were referred to compare the clinical efficacy of the two groups after treatment. Markedly effective: after treatment, the symptoms of gum pain, redness, and swelling of patients completely disappeared, how much tooth looseness decreased the I to II degree; Effective: after treatment, gum pain completely disappeared, redness and swelling symptoms disappeared, gum atrophy slightly improved, tooth looseness significantly relieved; ineffective: after treatment, gum pain, redness, and swelling symptoms, and tooth looseness, did not improve significantly or even worsened. Total effective rate $=$ (markedly effective + effective) $\times$ number of cases/total cases $\times 100 \%$. (II) Periodontal indexes: periodontal indexes in two groups before and after treatment were examined with a periodontal probe, including plaque index (PLI), periodontal pocket depth (PD), sulcus bleeding index (SBI), gingival index (GL) and clinical attachment loss (CAL). (III) Detection of CRP, TNF- $\alpha$, and IL- 6 levels in serum: $5 \mathrm{~mL}$ of fasting morning venous blood was aseptically collected from the two groups before and after treatment, centrifuged, and the serum samples were separated and stored at low temperature for examination. The CRP level in serum was detected by immunoturbidimetry, and the double-antibody sandwich ELISA method detected TNF- $\alpha$ and IL-6 levels in serum. The follow-up was one year, and the recurrence rate was counted for both groups.

\section{Statistical analysis}

Statistical software SPSS 20.0 was used for statistical analysis. The measurement data were expressed by mean \pm standard deviation (SD), the differences between groups or within groups were compared by $t$-test; the counting data were expressed by frequency (percentage), and the Mann-Whitney U rank-sum test compared the differences between groups of the counting data. $\mathrm{P}<0.05$ shows that the difference is statistically significant.

\section{Results}

\section{Comparison of periodontal indexes between the two groups}

The results in Figure 1 and Table 1 showed that, compared with the pre-treatment period, the PLI, SBI, PD, GL, and CAL in both groups after treatment significantly declined, and masticatory efficiency significantly increased $(\mathrm{P}<0.05)$. There are statistical differences in different indexes between the two groups before and after treatment $(\mathrm{P}<0.05)$. The difference between the control group and the experimental 
Table 1 Comparison of masticatory function scores between the two groups before and after treatment

\begin{tabular}{lcc}
\hline Group & Before treatment & After treatment \\
\hline Control group $(\mathrm{n}=48)$ & $0.68 \pm 0.05$ & $0.73 \pm 0.05$ \\
Experimental group $(\mathrm{n}=48)$ & $0.65 \pm 0.07$ & $0.85 \pm 0.07$ \\
$\mathrm{t}_{\text {within groups }}$ & 1.856 & 9.976 \\
$\mathrm{P}$ & 0.067 & 0.000 \\
$\mathrm{t}_{\text {between groups }}$ & \multicolumn{2}{c}{10.455} \\
$\mathrm{P}$ & \multicolumn{2}{c}{$<0.001$} \\
\hline
\end{tabular}

Values are mean \pm SD.

group was statistically significant, and the reduction in different periodontal indexes was significantly higher in the experimental group than in the control group after treatment. The increase of masticatory efficiency in the experimental group was significantly higher than that in the control group $(\mathrm{P}<0.05)$.

\section{Comparison of CRP, TNF- $\alpha$ and IL-6 levels in serum between the two groups}

The results in Figure 2 show that compared with the pre-treatment period, the levels of CRP, TNF- $\alpha$, and IL-6 in serum in both groups of patients after treatment were significantly reduced $(\mathrm{P}<0.05)$. After treatment, the reduction in TNF- $\alpha$ and IL-6 levels were significantly higher in the experimental group than in the control group $(\mathrm{P}<0.05)$, while although the expression of CRP in the experimental group was lower than that in the control group, there was no significant difference.

\section{Comparison of clinical efficacy between the two groups}

In terms of therapeutic effect (Table 2), the total effective rate of the experimental group was $95.83 \%$, while the control group was $83.33 \%$. Compared with the control group, the total effective rate of the experimental group increased significantly after treatment, and the difference was statistically significant $(\mathrm{P}<0.05)$.

\section{Comparison of follow-up between the two groups}

Patients in both groups were followed up for one year, with three cases of recurrence in each group and a recurrence rate of $6.25 \%$. The difference was not statistically significant
$(\mathrm{P}>0.05)$, comparing the two groups' recurrence rates.

\section{Discussion}

Periodontitis is often caused by plaque or calculus, and plaque microorganisms of the gingival crevice and their products cause inflammation and swelling of gums, leading the reproduction of some anaerobic bacteria, periodontal pathogens. With further development of periodontal inflammation, the periodontal membrane will be destroyed, leading to the resorption of alveolar bone, and the separation between the gingiva and dental root, thus causing the gingival sulcus, constantly deepens and forms periodontal pockets (8). When all the periodontal tissue is destroyed, the patients' teeth will become loose and displaced, resulting in a weak bite and bleeding gums and other typical symptoms $(9,10)$. Localized aggressive periodontitis is a severe type of periodontitis, as the disease progresses, periodontal tissue will be severely damaged. However, patients often ignore the disease in its early stages. Therefore, most of them have already experienced symptoms including looseness, displacement or loss of teeth by the time they visit the clinic, which has had a severe impact on their daily behaviors including chewing and vocalization, and posing difficulties and challenges for clinical diagnosis and treatment $(11,12)$. Localized aggressive periodontitis has a high incidence in clinical practice, usually caused by a mixed infection of many kinds of anaerobic bacteria (13). At present, it is considered that actinobacillus actinomycetemcomitans, meganucleus fusiform bacillus, mucosal actinomycetes are the primary pathogens of localized aggressive periodontitis, and the interaction between flora and specific host defense function further leads to periodontal tissue destruction, which mainly characterized by co-infection of attached plaque and bacteria of periodontal pockets (14). Conventional treatment of periodontitis mainly takes mechanical therapy, but it can only remove attached plaque, therefore, the application of antibacterial agents is also needed to control the related bacteria that cause the occurrence and development of the disease (15).

xipayi mouth rinse, a Uyghur medicine formula, mainly contains a Turkish gall whose principal component is tannin, a type of complex polyphenolic compound existing in nature and has effects including astringing lungs, invigorating qi, stopping bleeding and many others $(16,17)$. The clinical application effect of xipayi mouth rinse has been widely recognized, and it has shown a beneficial 



Figure 2 Comparison of inflammatory responses between the two groups before and after treatment $(\bar{x} \pm s) .{ }^{* *}, \mathrm{P}<0.01$. ns, no significant difference.

Table 2 Comparison of clinical therapeutic effects between the two groups

\begin{tabular}{lccc}
\hline Group & Markedly effective & Effective & Ineffective \\
\hline Control group $(\mathrm{n}=48)$ & $15(31.25)$ & $25(52.08)$ & $8(16.67)$ \\
Experimental group $(\mathrm{n}=48)$ & $26(54.17)$ & $20(41.67)$ & $2(4.17)$ \\
\hline
\end{tabular}

Values are presented as $n(\%)$.

effect in the treatment of periodontitis, gingivitis, and oral ulcers, including a significant anti-inflammatory effect and continuous bacteriostatic effect (18). xipayi mouth rinse also plays a unique role in regulating the balance of oral flora (18). Related pharmacological studies have shown xipayi mouth rinse can activate polymorphonuclear neutrophils (PMNs) and inhibit the formation of hydroxyl radicals, superoxide anion $\mathrm{O}_{2}$, and hydrogen peroxide, thus significantly relieving inflammatory responses including capillary exudation and edema, maintaining excellent oral status and enhancing therapeutic effects $(19,20)$. The pharmacological effect of minocycline hydrochloride mainly is it has a broad-spectrum antimicrobial effect on porphyromonas gingivalis and Capnocytophaga gingivalis $(21,22)$. Its mechanism of action is to inhibit bacterial protein synthesis and exert its antibacterial and antimicrobial effect, which can effectively inhibit the growth of gram-positive anaerobic bacteria and aerobes, gram-negative anaerobic bacteria and aerobic bacteria, and release the antimicrobial drugs slowly and continuously, and cause the tooth surface attached to connective tissue and fibroblasts again $(21,22)$. The affinity to the tooth surface is high, which can significantly reduce the adverse reactions $(21,22)$. Minocycline is an essential drug to treat aggressive periodontitis, but as a broad-spectrum antibiotic, it may destroy the balance of microorganisms in the oral environment and cause flora imbalance $(23,24)$. However, xipayi mouth rinse can make up for this deficiency $(23,24)$. The results of the present study suggest that combination drug therapy has a more pronounced inhibitory effect on localized aggressive periodontitis.

TNF- $\alpha$ is a multifunctional cytokine produced by activated monocytes and macrophages, which not only has an anti-tumor effect but also takes part in regulating immune response, substance metabolism, inflammatory response, among many others (25). It has been determined that TNF- $\alpha$ in the simple chronic periodontitis group is higher than that in the healthy group. These findings have suggested that TNF- $\alpha$ can activate osteoclasts and stimulate bone resorption. Furthermore, it can also stimulate the formation of osteoclast-like cells and dissolve and destroy connective tissue by inducing non-inflammatory cells, including prostaglandin E2 and metalloproteinases as mediators. It plays a vital role in alveolar bone resorption, periodontal connective tissue destruction, and periodontal attachment loss $(25,26)$. The endotoxin produced by G-anaerobes during periodontitis can also stimulate the synthesis and secretion of cytokines, including TNF- $\alpha$, reflected in the increase of TNF- $\alpha$ concentration in local periodontal tissue and gingival crevicular fluid, as well as in the peripheral blood of patients (27). Animal experimental studies also revealed that subcutaneous injection of periodontal pathogen toxins into hamsters could increase the level of inflammatory cytokines, including TNF- $\alpha$ in serum (28). The results of this study showed that TNF- $\alpha$ was significantly elevated in localized aggressive periodontitis, indicating that TNF- $\alpha$ has played a part in promoting inflammation in the disease. Concurrently, the expression level of TNF- $\alpha$ dropped significantly after treatment with xipayi mouth rinse combined minocycline. 
IL-6 is also a multifunctional cytokine with various biological activities. It can regulate immunity, take part in bone resorption, promote the proliferation of various cells $(29,30)$. It has been shown the content of IL-6 in periodontal tissue and serum are significantly higher in patients with periodontitis than patients in a normal situation, and their levels are positively correlated with the severity of periodontal lesions (31). Futrakul et al. (32) conducted a controlled study of patients with adult periodontal disease and periodontally healthy subjects and found the levels of cytokines TNF- $\alpha$ and IL- 6 produced by neutrophils in the oral and peripheral blood increased. Many studies have confirmed TNF- $\alpha$ and IL- 6 may facilitate oxidative stress, lead to continuous production of lipoperoxides, produce many toxic factors, cause vascular endothelial damage, change nitrous oxide products, and interfere with prostaglandin balance (33). CRP is a protein that appears in the acute phase of infectious or non-infectious inflammatory diseases (34). CRP not only serves as a marker of inflammation but also directly takes part in the inflammatory process itself (35). CRP cannot only stimulate monocytes to release inflammatory mediators, including TNF- $\alpha$, IL- 6 , and IL- $1 \beta$, but also stimulate vascular endothelial cells to up-regulate the expression of adhesive factors and induce the expression of proinflammatory cytokines in endothelial cells. Herrera and other studies found the content of CRP in the serum of women with chronic periodontitis elevated, which was negatively correlated with the prognosis (36). In this study, compared with the control group, the total effective rate of the drug combined treatment group significantly went up, the level of inflammatory factors IL-6 and CRP were significantly dropped, and the periodontal pocket depth, gingival index, and looseness were significantly improved. These results have shown xipayi mouth rinse combined with minocycline in the treatment of localized aggressive periodontitis can improve periodontal, gingival, and tooth looseness, and has a better clinical effect.

To sum up, the periodontitis symptoms of patients with localized aggressive periodontitis treated with xipayi mouth rinse combined with minocycline were improved, PLI, SBI, PD, GL, and CAL were significantly declined, and masticatory efficiency was significantly elevated. Inflammatory factors TNF- $\alpha$, IL-6, and CRP in serum also decreased, showing that xipayi mouth rinse combined with minocycline in the treatment of localized aggressive periodontitis can significantly improve the therapeutic effect and improve the gingival index and periodontal pocket depth. This study has supplied a theoretical and experimental basis to treat localized aggressive periodontitis.

\section{Acknowledgments}

We thank Guangzhou Yujia Biotechnology Co., Ltd for helpful conversations.

Funding: This work was supported by Hebei Province Medical Science Research Key Project Plan (project number: 20191812).

\section{Footnote}

Reporting Checklist: The authors have completed the CONSORT reporting checklist. Available at http://dx.doi. org/10.21037/apm-20-1653

Data Sharing Statement: Available at http://dx.doi. org/10.21037/apm-20-1653

Conflicts of Interest: All authors have completed the ICMJE uniform disclosure form (available at http://dx.doi. org/10.21037/apm-20-1653). The authors have no conflicts of interest to declare.

Ethical Statement: The authors are accountable for all aspects of the work in ensuring that questions related to the accuracy or integrity of any part of the work are appropriately investigated and resolved. All procedures performed in this study involving human participants were in accordance with the Declaration of Helsinki (as revised in 2013). The study was approved by the Ethics Committee of Handan Stomatological Hospital and informed consent was taken from all the patients.

Open Access Statement: This is an Open Access article distributed in accordance with the Creative Commons Attribution-NonCommercial-NoDerivs 4.0 International License (CC BY-NC-ND 4.0), which permits the noncommercial replication and distribution of the article with the strict proviso that no changes or edits are made and the original work is properly cited (including links to both the formal publication through the relevant DOI and the license). See: https://creativecommons.org/licenses/by-nc-nd/4.0/.

\section{References}

1. Allin N, Cruzalmeida Y, Velsko IM, et al. Inflammatory 
Response Influences Treatment of Localized Aggressive Periodontitis. J Dent Res 2016;95:635-41.

2. Miller K, Treloar T, Guelmann M, et al. Clinical Characteristics of Localized Aggressive Periodontitis in Primary Dentition. J Clin Pediatr Dent 2018;42:95-102.

3. Sase T. A case report of comprehensive treatment for a patient with localized aggressive periodontitis. Journal of The Japanese Society of Periodontology 2019;61:28-36.

4. Novaes AB Jr, Schwartz-Filho HO, de Oliveira RR, et al. Antimicrobial photodynamic therapy in the non-surgical treatment of aggressive periodontitis: microbiological profile. Lasers Med Sci 2012;27:389-95.

5. Guerrero A, Griffiths GS, Nibali L, et al. Adjunctive benefits of systemic amoxicillin and metronidazole in nonsurgical treatment of generalized aggressive periodontitis: a randomized placebo-controlled clinical trial. J Clin Periodontol 2005;32:1096-107.

6. Miller KA, Branco-de-Almeida LS, Wolf S, et al. Longterm clinical response to treatment and maintenance of localized aggressive periodontitis: a cohort study. J Clin Periodontol 2017;44:158-68.

7. Armitage GC. Development of a classification system for periodontal diseases and conditions. Ann Periodontol 1999;4:1-6.

8. Tonetti MS, Henry G, Kornman KS. Staging and grading of periodontitis: Framework and proposal of a new classification and case definition. J Periodontol 2018;89 Suppl 1:S159-72.

9. Papapanou PN, Mariano S, Nurcan B, et al. Periodontitis: Consensus report of workgroup 2 of the 2017 World Workshop on the Classification of Periodontal and Peri-Implant Diseases and Conditions. J Periodontol 2018;89:S173-82.

10. Kaneko C, Kobayashi T, Ito S, et al. Circulating levels of carbamylated protein and neutrophil extracellular traps are associated with periodontitis severity in patients with rheumatoid arthritis: A pilot case-control study. PLoS One 2018;13:e192365.

11. Harris TH, Wallace MR, Huang H, et al. Association of P2RX7 functional variants with localized aggressive periodontitis. J Periodontal Res 2020;55:32-40.

12. Zambon JJ. What happened to localized aggressive periodontitis? J Am Dent Assoc 2019;150:931-2.

13. Prud'homme T, Roy E, Soueidan A, et al. Multidisciplinary Clinical Management of a Localized Aggressive Periodontitis diagnosed in a Child with Glanzmann's Thrombasthenia. Int J Clin Pediatr Dent 2018;11:344-8.

14. Shaddox LM, Mullersman AF, Huang H, et al. Epigenetic regulation of inflammation in localized aggressive periodontitis. Clin Epigenetics 2017;9:94.

15. Shaddox LM, Spencer WP, Velsko IM, et al. Localized aggressive periodontitis immune response to healthy and diseased subgingival plaque. J Clin Periodontol 2016;43:746-53.

16. Aibibai Y, Hamulati W, Zhong LJ. Effects of xipayi mouth rinse on the DNA synthesis and change of cell cycles of human gingival fibroblast induced by lipopolysaccharide. Zhong Nan Da Xue Xue Bao Yi Xue Ban 2006;31:483-6.

17. Yan L, Wu PL. Research status of Xipayi mouth rinse used in treatment of oral diseases. Chinese Journal of Conservative Dentistry 2009;19:489-91.

18. Chen F, Jiao P, Che H, et al. Improvement effect of Shuanghuangbu combined with Xipayi Mouth Rinse on periodontal inflammation of peri-implantitis patients and its clinical significance. Journal of Jilin University 2017;43:1226-30.

19. Zuomi W. Effect of Xipayi mouth rinse on gingival bleeding caused by gingivitis. Beijing Journal of Stomatology 2010.

20. Hong T, Stomatology DO. The effect of Xipayi mouth rinse fluid combined with Kouyanqing grains on recurrent aphthous ulcer. Journal of Practical Stomatology 2014;30:431-3.

21. Lu HK, Chei C. Efficacy of subgingivally applied minocycline in the treatment of chronic periodontitis. J Periodontal Res 2005;40:20-7.

22. Wang X, Ma J, Zhu X, et al. Minocycline-loaded in situ hydrogel for periodontitis treatment. Curr Drug Deliv 2018;15:664-71.

23. Zhou J. Analysis of efficacy of minocycline hydrochloride ointment in treating aggressive periodontitis. Jiangsu Medical Journal 2011;37:712-3.

24. Matthews BR, Brereton MF. Application of Minocycline HCL in Treatment of Periodontitis. Journal of Henan University of Science \& Technology (Medical Science) 2008;79:509-16.

25. Darvishi E, Aziziaram Z, Yari K, et al. Lack of association between the TNF- $\alpha-1031$ genotypes and generalized aggressive periodontitis disease. Cell Mol Biol (Noisy-legrand) 2016;62:63-6.

26. Bastos MF, Lima JA, Vieira PM, et al. TNF- $\alpha$ and IL-4 levels in generalized aggressive periodontitis subjects. Oral Dis 2009;15:82-7.

27. Fredriksson M, Bergström K, Asman B. IL- 8 and TNF- $\alpha$ from peripheral neutrophils and acute-phase proteins in periodontitis: effect of cigarette smoking: a pilot study. J 
Clin Periodontol 2002;29:123-8.

28. Hojo K, Tamura A, Mizoguchi C, et al. Predominant Bacteria Recovered from a Periodontitis Site in a Hamster Model Raised by Silk-Ligature with Prophyromonas gingivalis Infection. Biosci Biotechnol Biochem 2008;72:1348-51.

29. Hanai Y, Sugita N, Wang Y, et al. Relationships between IL-6 gene polymorphism, low BMD and periodontitis in postmenopausal women. Arch Oral Biol 2015;60:533-9.

30. Sharma M, Bairy I, Pai K, et al. Salivary IL-6 levels in oral leukoplakia with dysplasia and its clinical relevance to tobacco habits and periodontitis. Clin Oral Investig 2011;15:705-14.

31. Noh MK, Jung M, Kim SH, et al. Assessment of IL-6, IL- 8 and TNF- $\alpha$ levels in the gingival tissue of patients with periodontitis. Exp Ther Med 2013;6:847-51.

32. Futrakul N, Butthep P, Patumraj S, et al. Enhanced tumor necrosis factor in the serum and renal hypoperfusion in nephrosis associated with focal segmental

Cite this article as: Chen X, Wang L, He Z, Zhao H, Cai Y, Song S, Yu Q. Effects of xipayi mouth rinse combined with minocycline on localized aggressive periodontitis' therapeutic effect and the levels of CRP, TNF- $\alpha$, IL-6 in serum. Ann Palliat Med 2020;9(5):3410-3417. doi: 10.21037/apm-20-1653 glomerulosclerosis. Ren Fail 2000;22:213-7.

33. Ramirez-Ramirez V, Maciasislas MA, Ortiz GG, et al. Efficacy of Fish Oil on Serum of TNF $\alpha$, IL-1 $\beta$, and IL-6 Oxidative Stress Markers in Multiple Sclerosis Treated with Interferon Beta-1b. Oxid Med Cell Longev 2013;2013:709493.

34. Xia QR, Liang J, Cao Y, et al. Increased plasma nesfatin-1 levels may be associated with corticosterone, IL-6, and CRP levels in patients with major depressive disorder. Clin Chim Acta 2018;480:107-11.

35. Schmidt FM, Schr Der T, Kirkby KC, et al. Pro- and anti-inflammatory cytokines, but not CRP, are inversely correlated with severity and symptoms of major depression. Psychiatry Res 2016;239:85-91.

36. Herrera JA, Parra B, Herrera E, et al. Periodontal disease severity is related to high levels of C-reactive protein in pre-eclampsia. J Hypertens 2007;25:1459-64.

(English Language Editor: J. Chapnick) 